\title{
Repetition proportion affects masked priming in nonspeeded tasks
}

\author{
GLEN E. BODNER AND JEREMY C. S. JoHNSON \\ University of Calgary, Calgary, Alberta, Canada
}

\begin{abstract}
Masked repetition priming is often greater when a larger proportion of trials involve repetition primes, suggesting that a context-sensitive unconscious process may be operating. Two recent studies have failed to obtain an effect of prime proportion in the perceptual identification (PI) task, suggesting that the effect may not occur in nonspeeded tasks. Contrary to this possibility, we report proportion effects with masked repetition primes in two nonspeeded tasks: PI and fragment completion (FC). A proportion effect occurred in the accuracy measure only in the FC task, but it occurred in the reaction time measure in both tasks. Prior failures to find proportion effects in the PI task thus may have been due, in part, to the dependent measure used. We interpret our findings in light of several recent accounts of prime proportion effects with brief primes.
\end{abstract}

The masked priming paradigm is commonly used to evaluate how unconscious stimuli affect responses to consciously perceived targets. A short-duration prime (e.g., horse for $45 \mathrm{msec}$ ) is preceded by a premask (e.g., XXXXX for $495 \mathrm{msec}$ ) and is followed by a target (e.g., HORSE) to which the subject responds. Using this arrangement, Forster and Davis (1984) found that masked repetition primes (e.g., horse-HORSE) facilitated lexical decisions to word targets, relative to an unrelated-prime control condition (e.g., stair-HORSE). They suggested that this paradigm is useful for isolating the effects of "low-level" lexical processes from those of context-sensitive "high-level" processes (see also Forster, Mohan, \& Hector, 2003).

Recent masked priming studies in which the proportion of repetition (vs. unrelated) primes in the stimulus list (i.e., $R P$ ) was manipulated appear to challenge this conclusion. If masking primes eliminates context-sensitive higher level processes, responses should not be sensitive to the list-wide RP. Contrary to this possibility, several lexical decision studies have reported that facilitation of reaction times (RTs) from repetition primes is greater when RP is high rather than low (e.g., Bodner \& Masson, 2001; Bodner, Masson, $\&$ Richard, 2006; Bodner \& Stalinski, 2008), and analogous proportion effects have now been obtained with a variety of stimuli in a variety of tasks (Bodner \& Dypvik, 2005; Bodner \& Masson, 2004; Jaskowski, Skalska, \& Verleger, 2003; Kinoshita, Forster, \& Mozer, 2008; Klapp, 2007).

These influences of prime proportion on masked priming indicate that either the process that produces masked priming or some aspect of the decision process is context sensitive. In Forster and Davis's (1984) account, masked priming reflects the automatic process of opening of a lexical entry; hence, proportion effects on masked priming must be attributed to the decision stage. Kinoshita and her colleagues (e.g., Kinoshita et al., 2008) have recently posited an adaptation to the statistics of the environment (ASE) account that builds on Lupker, Brown, and Colombo's (1997) suggestion that when performing a speeded task, subjects attempt to set a response deadline that will allow them to do so while minimizing their errors.

In the ASE account, the deadline established on a given trial is based on the system's attempt to minimize the combination of its estimated error rate and the cost it associates with taking longer to respond. The estimated error rate is based on a combination of the difficulty of target processing on the current trial and on recent trials. This error estimate decreases over time as decision evidence accumulates, and it decreases more rapidly for "easy" repetition-primed trials than for "hard" unrelated-primed trials. In contexts in which there are more easy trials than difficult trials, such as in a high-RP context, the estimated error rate for the easy trials decreases more rapidly than in a low-RP context, but it does not change much for the hard trials. This difference produces elevated priming in the high-RP group (see Kinoshita et al., 2008).

The ASE account provides a well-specified alternative to Bodner and Masson's memory recruitment account of proportion effects (e.g., Bodner \& Masson, 2001; Masson \& Bodner, 2003). According to the latter account, the processing performed on a masked prime is encoded, and this processing will be recruited to help subjects identify or classify a target if the context supports this recruitment. A high RP provides such a supportive context. The memory recruitment account uses the same mechanismrecruitment of prime processing - to explain both priming and prime proportion effects. Thus, according to the memory recruitment account, the cognitive system attunes to the usefulness of the masked primes for performing the

G. E. Bodner, bodner@ucalgary.ca 
target task, whereas according to the ASE account, it attunes to current and recent trial difficulty. Kinoshita et al. (2008) suggested that the latter attunement is "dumber" and is, thus, in better keeping with the conceptualization of unconscious processes vis-à-vis conscious processes.

The ASE and memory recruitment accounts make different predictions regarding whether prime proportion should affect priming in nonspeeded tasks. As was stated by Kinoshita et al. (2008), "When the task does not require speeded responding ... there is no basis to expect this adaptation to the stimulus environment to occur, hence no proportion effect is expected" (p. 644). In contrast, according to the memory recruitment account, the usefulness of the prime context should modulate recruitment of the primes regardless of whether the task is speeded.

To date, two studies have looked for prime proportion effects with masked primes in a nonspeeded perceptual identification (PI) task (Evett \& Humphreys, 1981). Each trial in the PI task consists of a premask, a brief lowercase prime, a brief uppercase target, and a postmask. The subject tries to identify the target. The instructions place no emphasis on speeded responding; hence, a response deadline should not be imposed. Using Dutch stimuli, Brysbaert (2001, Experiment 3) found equivalent homophonic priming (e.g., bear-BARE vs. two-BARE) whether .72 or .14 of the trials had homophonic primes. Pecher, Zeelenberg, and Raaijmakers (2002, Experiment 2), also using Dutch stimuli, found equivalent masked associative priming (e.g., salt-PEPPER vs. tiger-PEPPER) whether .9 or .1 of the trials had associative primes.

As was noted by Kinoshita et al. (2008), the absence of proportion effects in the nonspeeded PI task is in keeping with the ASE account, but it challenges the memory recruitment account. However, it is unclear whether these null effects highlight an important dissociation between speeded and nonspeeded tasks, or whether they were due to some aspect of the design/procedure that differed from prior tests of prime proportion effects in speeded tasks (e.g., prime type, language). Given that our lab has often reported proportion effects on masked repetition priming in English, the simple approach we adopted here was to test whether RP affects priming in nonspeeded identification tasks with English stimuli.

In Experiment 1, we manipulated RP in the PI task. In addition to measuring accuracy, we also measured RTs, because prior RP effects from our lab have typically been more robust - or were only present - in the RT measure. Brysbaert (2001) and Pecher et al. (2002) did not measure RTs; thus, it is possible that their failure to find proportion effects was due to their exclusive reliance on accuracy as the dependent measure. In Experiment 2, we examined a second nonspeeded task.

\section{EXPERIMENT 1 Perceptual Identification}

In Experiment 1, across groups we manipulated whether 20 or 80 of 100 trials in the PI task involved repetition (vs. unrelated) primes. We measured both accuracy and RT.

\section{Method}

Subjects. University of Calgary undergraduates, 50 in all, participated for extra course credit. They were randomly assigned to the high- or low-RP group (25 each).

Materials and Design. The critical targets were 100 words, five letters in length, that ranged in frequency from 0 to 198 per million (median $=14 ;$ Kučera \& Francis, 1967). Each target was paired with a unique unrelated prime, also five letters in length and of similar frequency ( 0 to 204 ; median $=14$ ), which shared no more than two letters with the target and in which no letters that were shared were in the same position as in the target (e.g., evoke-LINEN, sound-PEACE). An additional 10 target/unrelated-prime pairs were used for practice trials. The RP on practice trials matched that on critical trials. In the high-RP group, 80 of the 100 trials had repetition primes and 20 had unrelated primes, and vice versa for the low-RP group. All the subjects received the same target set. The assignment of repetition or unrelated primes was counterbalanced, so that, across all subjects, each target was paired with each type of prime equally often.

Procedure. The subjects were tested individually using a Macintosh computer. The stimuli were presented in black 12-point Courier font on a white background. The subjects were told that they would be shown a series of trials, each involving a brief uppercase target; they were not specifically informed about the primes. Each trial consisted of a 495-msec premask (XXXXX), followed immediately by a 45 -msec prime in lowercase, followed immediately by a 45 -msec target in uppercase, followed immediately by a postmask (@@@@@) that remained in view until the subject responded.The subjects pressed the " 1 " key on the keyboard with their left index finger if they thought that they could identify the target; a text box then appeared on the screen, into which they typed their response. The subjects pressed the " 6 " key on the keyboard with their right index finger if they could not identify the target. RTs were measured from the onset of the target until a keypress. The subjects were instructed to return their index fingers to the " 1 " and " 6 " keys after keying in their previous response. The intertrial interval was $1 \mathrm{sec}$. The instructions did not mention speed or accuracy of responding, and feedback regarding speed and accuracy was not provided. The 10 randomized practice trials were presented followed by the 100 randomized critical trials. A brief rest break was provided after 50 critical trials.

\section{Results and Discussion}

An accurate response was defined as responding with the target (although the results did not change when responses that were either one or two letters different from the target were considered accurate). Trials with RTs shorter than $300 \mathrm{msec}$ were considered errors and were excluded from analysis (1.6\%). We set our upper RT cutoff so that just fewer than $1.0 \%$ of the trials were excluded in each experiment, resulting in a 2.6-sec upper RT cutoff in Experiment 1. Variability in RTs was substantial because our instructions did not emphasize speed. We therefore computed median RTs on the basis of all accurate responses on critical trials, the means of which are shown in Figure 1, along with the mean accuracy rates. An ANOVA with RP group (high, low) as the betweensubjects factor and prime type (repetition, unrelated) as the within-subjects factor was computed for each measure. Effects were significant at the .05 level unless otherwise noted.

Overall, accuracy and RT did not differ across the two RP groups $\left(F_{\mathrm{s}}<1\right)$. Robust masked priming effects occurred in each measure: The subjects were $31 \%$ more accurate following repetition primes than following unre- 


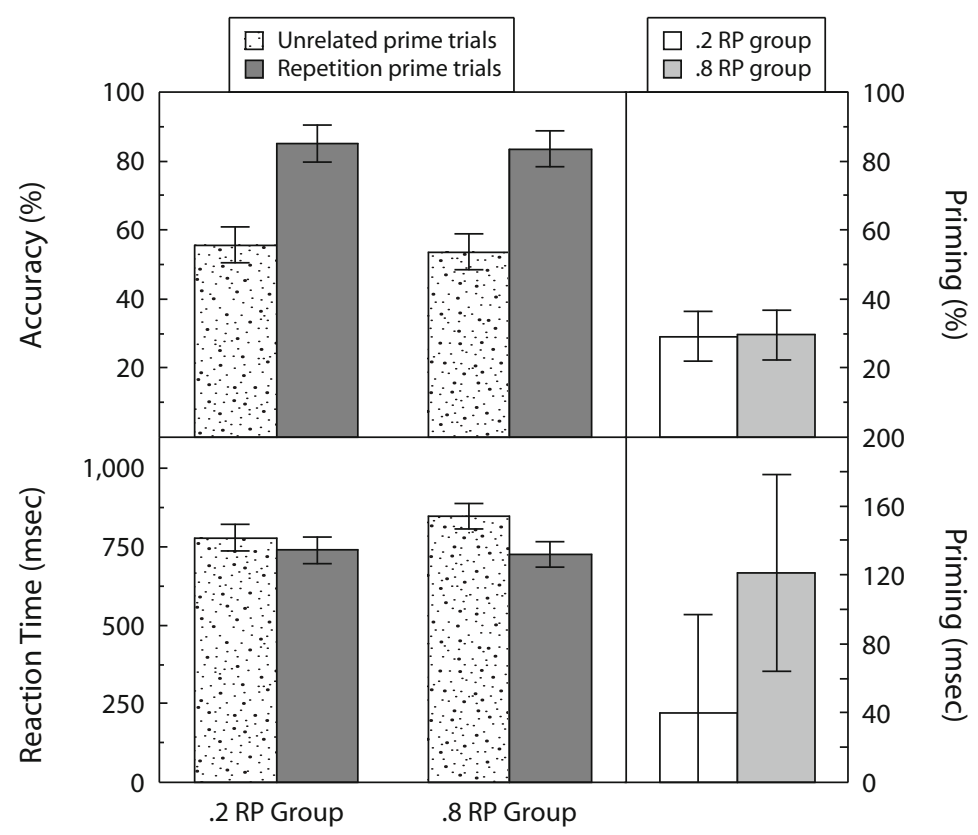

Figure 1. Experiment 1: Perceptual identification results. In the accuracy and reaction time panels, the $95 \%$ within-subjects confidence intervals show whether priming occurred in each repetition proportion (RP) group. In the priming panels, the $95 \%$ between-subjects confidence intervals show whether priming differed across the RP groups.

lated primes $\left[F(1,48)=137.64, M S_{\mathrm{e}}=170\right]$ and were also $81 \mathrm{msec}$ faster $\left[F(1,48)=16.29, M S_{\mathrm{e}}=10,045\right]$. Turning to the key interactions, the effect of priming on accuracy was the same in the high- and low-RP groups $(31 \%$ vs. $31 \% ; F<1)$, replicating prior failures to find a proportion effect in the PI task (Brysbaert, 2001; Pecher et al., 2002). However, the repetition priming effect on RTs - which was not measured in those studies - was three times larger in the high-RP group than in the lowRP group [122 vs. $40 \mathrm{msec} ; F(1,48)=4.11, M S_{\mathrm{e}}=$ $10,045]$. Priming was significant when the RP was high $\left[F(1,24)=20.28, M S_{\mathrm{e}}=9,108\right]$, but not when the RP was low $\left[F(1,24)=1.84, M S_{\mathrm{e}}=10,981, p=.19\right]$.

The large priming effects we observed could perhaps have come about because of "double-dipping": On repetition trials, the subjects could respond either with the prime or with the target, whereas on unrelated trials, only responding with the target was considered accurate. However, responding with the unrelated prime was quite rare and did not differ between the high- and low-RP groups $\left[2.2 \%\right.$ vs. $\left.3.9 \% ; F(1,48)=1.06, M S_{\mathrm{e}}=33.9, p=.31\right]$. Thus, double-dipping does not account for the large priming effect or the RP effect, and the low rate of responding with the unrelated prime also suggests that our primes were effectively masked.

In sum, in agreement with previous findings (Brysbaert, 2001; Pecher et al., 2002), accuracy in the PI task was not affected by masked prime proportion. The novel outcome of Experiment 1 was that a robust RP effect was observed in the RTs, even though our instructions did not request speeded responses. An RP effect can thus be observed in a nonspeeded task, at least in the RTs. This result was not predicted by the ASE account, but it was predicted by the memory recruitment account. Before pursuing these implications, however, we examined whether the RP effect could be replicated in another nonspeeded task.

\section{EXPERIMENT 2 Fragment Completion}

The goal of Experiment 2 was to test whether RP would affect priming in a fragment completion (FC) task in which each masked prime was followed by a word fragment allowing at least two completions. On repetition prime trials, the designated target completion served as the prime (e.g., house-H\&\&SE), whereas on unrelated-prime trials an unrelated word served as the prime (e.g., stair-H\&\&SE). The subjects completed each target fragment with the first word that came to mind. As in Experiment 1, our instructions did not mention speeded responding. Forster, Booker, Schacter, and Davis (1990) reported sizable masked repetition priming effects on accuracy with this paradigm.

\section{Method}

Subjects. Fifty new subjects from the same University of Calgary pool participated. Half were randomly assigned to each RP group.

Materials, Design, and Procedure. This experiment differed from Experiment 1 in only two respects. First, a postmask was not presented. Instead, after the 45-msec prime, the target remained on the screen until a response was made. These trial specifications pro- 


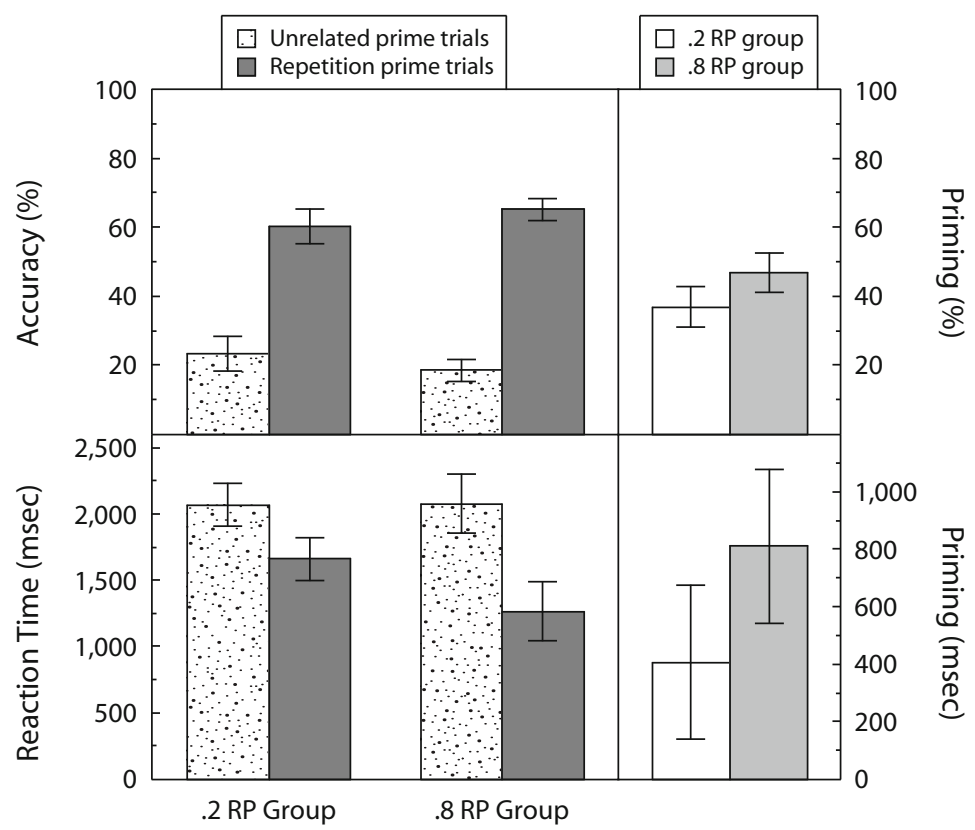

Figure 2. Experiment 2: Fragment completion results. In the accuracy and reaction time panels, the $95 \%$ within-subjects confidence intervals show whether priming occurred in each repetition proportion (RP) group. In the priming panels, the $95 \%$ between-subjects confidence intervals show whether priming differed across the RP groups.

duce a minimal prime awareness that does not modulate the size of RP effects (e.g., Bodner \& Masson, 2004; Bodner et al., 2006; Bodner \& Stalinski, 2008). Second, two letters of the Experiment 1 targets (e.g., HOUSE) were replaced by ampersands (e.g., H\&\&SE), so that at least one nontarget completion was possible (e.g., HORSE). The subjects were asked to complete each target word fragment with the first five-letter completion that came to mind that was not a plural. Speed of responding was not mentioned.

\section{Results and Discussion}

An accurate response was defined as responding with the designated target completion (e.g., HOUSE, but not HORSE, for H\&\&SE). Trials with RTs shorter than $300 \mathrm{msec}$ $(0.1 \%)$ or longer than $9 \mathrm{sec}(1.0 \%)$ were excluded. The mean of the subjects' median RTs for accurate responses and their mean accuracy in each priming condition appear in Figure 2. Overall, accuracy and RT did not differ across RP group $\left(F_{\mathrm{s}}<1.25\right)$. Robust repetition priming occurred: The subjects were $42 \%$ more accurate following repetition primes than following unrelated primes $\left[F(1,48)=413.95, M S_{\mathrm{e}}=106\right]$ and were also $610 \mathrm{msec}$ faster $\left[F(1,48)=42.02, M S_{\mathrm{e}}=221,358\right]$. In agreement with Experiment 1, the priming effect on RTs was far larger in the high-RP group than in the low-RP group [812 vs. $\left.408 \mathrm{msec} ; F(1,48)=4.62, M S_{\mathrm{e}}=221,358\right]$. Most important, we also found a reliable RP effect on accuracy - an effect not previously found using the identification version of the PI task (Experiment 1; Brysbaert, 2001; Pecher et al., 2002). The priming effect was $47 \%$ in the high-RP group, as compared with $37 \%$ in the low-RP group $\left[F(1,48)=5.36, M S_{\mathrm{e}}=106\right]$. The priming effect on each measure within each RP group was consistently significant $(p \mathrm{~s}<.01)$.

\section{GENERAL DISCUSSION}

We evaluated the mechanism responsible for prime proportion effects on masked repetition priming by more closely examining whether RP effects occur in nonspeeded tasks. Two previous studies using the PI task (Brysbaert, 2001; Pecher et al., 2002) suggested that prime proportion effects with masked primes are absent in nonspeeded identification tasks. However, we found greater masked repetition priming effects when the RP was larger in both the PI task (in the RT measure) and the FC task (in both the RT and accuracy measures).

Recently, Weidemann, Huber, and Shiffrin (2008) reported prime proportion effects on accuracy with brief but unmasked primes in a variant of the PI task. Although their effect is analogous to ours, they intermixed trials of long- and short-duration primes, and they used a forced choice variant of the PI task. Weidemann et al. interpreted their "prime diagnosticity effects" using a formal Bayesian model called responding optimally with unknown sources of evidence (ROUSE). According to ROUSE, higher proportion groups are less likely to discount evidence provided by the brief primes, which is equivalent to the memory recruitment account's claim that higher proportion groups are more likely to rely on evidence provided by brief primes. In the ROUSE model, prime and target percepts are first integrated and then compared 
with existing feature vectors in memory, whereas in the memory recruitment account, target processing cues retrieval of prior episodes from memory, including the episode formed for the prime processing. Therefore, the occurrence of nonword priming in an all-nonword variant of their paradigm would be compatible with the memory recruitment account, but not with the ROUSE account. In addition, it remains to be seen whether proportion effects will occur in an identification (cf. forced choice) version of their paradigm, or in a version that uses only shortduration masked primes. Discounting of prime evidence may be more likely to occur when long-prime-duration trials are included and when the target task involves making forced choices between two orthographically dissimilar targets.

The occurrence of prime proportion effects on accuracy in nonspeeded tasks here and in Weidemann et al. (2008) is not predicted by the ASE account. Of course, our finding that RP affected priming in RTs could mean that the subjects adopted response deadlines in these experiments despite not being given instructions to respond quickly. After all, subjects must choose a point in time to respond in any task, and in this sense, every task ultimately requires subjects to impose a response deadline. Nonetheless, the memory recruitment account remains consistent with RP effects in both "speeded" and "nonspeeded" tasks, whereas the ASE account cannot appeal to a dissociation between these two types of task as evidence in its favor.

Both the ASE and memory recruitment accounts face other challenges. For the ASE account, proportion effects in tasks that do not involve response-incongruent unrelated-prime trials - particularly lexical decisionmay require postulation of a different mechanism (see Kinoshita et al., 2008, p. 645). For the memory recruitment account, Kinoshita et al.'s finding that the presence of response-incongruent primes can modulate proportion effects in the naming task (cf. the lexical decision task; Bodner et al., 2006) provides a new challenge. It is conceivable that some proportion effects arise at the stimulus-processing stage, whereas others arise at the decision stage, and that different mechanisms operate at each locus. Whether the proportion effect emerges in the accuracy or the RT measure could depend on this locus.

As Kinoshita et al. (2008) noted, delineating the conditions that predict when prime proportion effects will occur is an important ongoing process. With respect to the PI task, our experiments suggest that prior failures to find this effect in this task were due partly to exclusive use of accuracy as the dependent measure. In addition, Weidemann et al. (2008) reported that proportion effects can be sensitive to within-experiment shifts in prime proportion. In this connection, they suggested that Pecher et al. (2002) may have failed to find a proportion effect on accuracy in the PI task because both groups received a set of 60 threshold trials (for setting the masked prime duration) before the main experiment in which the primes were always unrelated to the target. However, we included no such trials, and we also did not obtain a proportion effect on accuracy in this task.

Finally, it remains unclear why RP affected accuracy in our FC task but not in our PI task. The accuracy measure in our PI task may simply have been a less sensitive measure than our accuracy measure in our FC task. It remains to be seen whether this is a replicable and important dissociation. ${ }^{1}$ The occurrence of prime proportion effects on accuracy in our WC task and in Weidemann et al.'s (2008) forced choice version of the PI task suggests that the absence of such effects in the identification version of the PI task (Brysbaert, 2001; Pecher et al., 2002) may be a consistent exception to the rule. Our findings suggest that researchers using priming tasks should always examine both accuracy and RT measures, even if their priming tasks are nonspeeded.

\section{AUTHOR NOTE}

The Natural Sciences and Engineering Research Council of Canada supported this research through a Discovery Grant to G.E.B. and through an Undergraduate Student Research Award to J.C.S.J. We thank Sachiko Kinoshita, Diane Pecher, Christoph Weidemann, and Rene Zeelenberg for their comments on an earlier version of the manuscript. Correspondence should be sent to G. E. Bodner, Department of Psychology, University of Calgary, 2500 University Drive NW, Calgary, AB, T2N 1N4 Canada (e-mail: bodner@ucalgary.ca).

\section{REFERENCES}

BoDNer, G. E., \& DYPVIK, A. T. (2005). Masked priming of number judgments depends on prime validity and task. Memory \& Cognition, 33, 29-47.

Bodner, G. E., \& Masson, M. E. J. (2001). Prime validity affects masked repetition priming: Evidence for an episodic resources account of priming. Journal of Memory \& Language, 45, 616-647.

Bodner, G. E., \& MAsson, M. E. J. (2004). Beyond binary judgments: Prime validity modulates masked repetition priming in the naming task. Memory \& Cognition, 32, 1-11.

Bodner, G. E., Masson, M. E. J., \& Richard, N. T. (2006). Repetition proportion biases masked priming of lexical decisions. Memory \& Cognition, 34, 1298-1311.

Bodner, G. E., \& Stalinski, S. M. (2008). Masked repetition priming and proportion effects under cognitive load. Canadian Journal of Experimental Psychology, 62, 127-131.

BrysbaERT, M. (2001). Prelexical phonological coding of visual words in Dutch: Automatic after all. Memory \& Cognition, 29, 765-773.

Evett, L. J., \& Humphreys, G. W. (1981). The use of abstract graphemic information in lexical access. Quarterly Journal of Experimental Psychology, 33A, 325-350.

Forster, K. I., Booker, J., Schacter, D. L., \& Davis, C. (1990). Masked repetition priming: Lexical activation or novel memory trace? Bulletin of the Psychonomic Society, 28, 341-345.

Forster, K. I., \& DAVIS, C. (1984). Repetition priming and frequency attenuation in lexical access. Journal of Experimental Psychology: Learning, Memory, \& Cognition, 10, 680-698.

Forster, K. I., Mohan, K., \& Hector, J. (2003). The mechanics of masked priming. In S. Kinoshita \& S. J. Lupker (Eds.), Masked priming: The state of the art (pp. 3-37). New York: Psychology Press.

Jaskowski, P., Skalska, B., \& Verleger, R. (2003). How the self controls its "automatic pilot" when processing subliminal information. Journal of Cognitive Neuroscience, 15, 1-10.

Kinoshita, S., Forster, K. I., \& Mozer, M. C. (2008). Unconscious cognition isn't that smart: Modulation of masked repetition priming effect in the naming task. Cognition, 107, 623-649.

KLAPP, S. T. (2007). Nonconscious control mimics a purposeful strategy: Strength of Stroop-like interference is automatically modulated 
by proportion of compatible trials. Journal of Experimental Psychology: Human Perception \& Performance, 33, 1366-1376.

KUČERA, H., \& FranCIS, W. N. (1967). Computational analysis of presentday American English. Providence, RI: Brown University Press.

LuPKer, S. J., Brown, P., \& Colombo, L. (1997). Strategic control in a naming task: Changing routes or changing deadlines? Journal of Experimental Psychology: Learning, Memory, \& Cognition, 23, 570-590.

Masson, M. E. J., \& Bodner, G. E. (2003). A retrospective view of masked priming: Toward a unified account of masked and long-term repetition priming. In S. Kinoshita \& S. J. Lupker (Eds.), Masked priming: The state of the art (pp. 57-94). New York: Psychology Press.

Pecher, D., Zeelenberg, R., \& RaAijmakers, J. G. W. (2002). Associative priming in a masked perceptual identification task: Evidence for automatic processes. Quarterly Journal of Experimental Psychology, 55A, 1157-1173.

Weidemann, C. T., Huber, D. E., \& Shiffrin, R. M. (2008). Prime diagnosticity in short-term repetition priming: Is primed evidence discounted, even when it reliably indicates the correct answer? Journal of Experimental Psychology: Learning, Memory, \& Cognition, 34, 257-281.

\section{NOTE}

1. We conducted an additional experiment $(N=80)$ using a hybrid of our two tasks. We took the PI task and made it more like the FC task by presenting the target for $90 \mathrm{msec}$ and by removing one letter from each target. The main findings were a replication of the RP effect on RTs [280 vs. $\left.74 \mathrm{msec} ; F(1,48)=8.47, M S_{\mathrm{e}}=47,983\right]$ and a small RP effect on accuracies ( $48 \%$ vs. $43 \%)$ that did not reach significance $\left[F(1,78)=2.29, M S_{\mathrm{e}}=113, p=.13\right]$. Interestingly, however, the $5 \%$ $\mathrm{RP}$ effect on accuracy was halfway between our $0 \%$ (PI task) and $10 \%$ (FC task) effects.

(Manuscript received August 11, 2008; revision accepted for publication December 4, 2008.) 